\title{
ESTRATEGIAS AGROALIMENTARIAS COMO HERRAMIENTA DE DISEÑO DE LOS SISTEMAS AGROALIMENTARIOS LOCALES
}

\author{
Estefanía Osorio-Acosta *a; Inmaculada Marqués-Pérez ${ }^{\mathrm{a}}$ y Baldomero Segura ${ }^{\mathrm{a}}$ \\ ${ }^{a}$ Universitat Politècnica de València. Valencia. imarques@ upvnet.upv.es
}

\begin{abstract}
Resumen
En los Sistemas agroalimentarios locales los alimentos son producidos, procesados y comercializados en un área geográfica definida, siendo fácilmente trazables desde su origen hasta su consumo. El sentido local se entiende desde la escala geográfica, por el efecto que tiene la proximidad sobre el bajo impacto ambiental del transporte, hasta la relación de confianza existente entre el consumidor y el productor. Para construir estos sistemas se requiere del diseño de estrategias específicas que permitan su desarrollo integrado. El objeto de este trabajo ha sido el de contribuir al proceso de definición de Estrategias Agroalimentarias Sostenibles de carácter Local (EASL). Para ello, tras la identificación de los retos alimentarios que deberá resolver el sistema se propuso las etapas que deberían incluir el proceso estratégico y las reflexiones sobre sus resultados.
\end{abstract}

\section{Palabras claves}

Estrategias agroalimentarias locales, sistemas agroalimentarios, agricultura periurbana, caso de estudio

\section{Introducción y objetivos}

Los sistemas de producción integrada, de producción ecológica o la transición hacia sistemas agroecológicos (Gliessman et al., 2007) de carácter peri-urbano (Paül y McKenzie, 2013); (Mincyte y Dobernig, 2016); (Cerrada-Serra et al., 2018) son muestra del amplio consenso que existe en torno a los cambios que deben producirse en el modelo de producción agraria para conseguir productos con menor impacto sobre el medioambiente y socialmente inclusivos (Hinrichs, 2000); (Donald y Blay-Palmer, 2006). No parece estar tan generalizada, sin embargo, la opinión sobre la distribución comercial, dado el reto que implica el desarrollo de un sistema logístico competitivo, especialmente allí donde existen grandes distancias entre los centros principales de consumo y los de producción (Brunori et al., 2016); (Rodríguez Guerra et al., 2017). Despunta así el llamado comercio de proximidad o cadena comercial corta cuyo objetivo es reconectar a los consumidores urbanos con los productores periurbanos y rurales en torno a valores relacionados con la soberanía alimentaria (Pribadi y Pauleit, 2015), seguridad alimentaria (Kneafsey et al., 2013) y la sostenibilidad (De Cunto et al., 2017). El comercio de proximidad representa un modelo alternativo de transacción económica (Opitz et al., 2017); (Zoll et al., 2018) que incluye distintas tipologías de redes alimentarias (Sánchez, 2009) organizadas en torno a la cadena de suministros (Renting et al., 2003).

La Organización de las Naciones Unidas para la Alimentación y la Agricultura (FAO, 2011); (FAO y OPS, 2016) recoge estos valores y prácticas al definir un Sistema de Alimentación Sostenible (SAS) como aquél que garantiza la seguridad alimentaria y de nutrición para todos, incluidas las futuras generaciones; pero evoluciona el enfoque al proponer la integración de los sistemas agroalimentarios en el territorio, basados en nuevas formas de conectividad entre lo urbano y lo rural, aportando al concepto de sostenibilidad una mayor y más profunda significancia al integrar salud humana y medioambiental (Marsden y Sonnino, 2012b). Surgen así los Sistemas de Alimentación Sostenibles y de carácter Local (SIAL) como alternativas que se centran en la preeminencia del territorio y de las redes locales o regionales. Su evolución y consolidación depende de acciones sociales reciprocas entre los elementos que intervienen para dotarlo de estabilidad y eficacia sistémica (Muchnik et al., 2008) de naturaleza multidimensional. La especificidad de cada SIAL depende de los recursos territoriales implicados, la configuración del sector agroalimentario, las estrategias organizacionales e institucionales que se movilizan por los actores (Boucher y Reyes González, 2016) (Torres-Salcido y Sanz-Cañada, 2018) y las singularidades presentes en su patrimonio natural y sociocultural (Rastoin, 2015); (Calori et al., 2017).

La escala territorial que se defina es importante a la hora de impulsar y desarrollar estos SIAL. El nivel municipal es el mínimo nivel competencial que permite la definición de Estrategias Agroalimentarias Locales Sostenibles (EALS). Estas estrategias pueden definirse como un proceso consistente en cómo una ciudad visualiza el cambio en su sistema agroalimentario y cómo se esfuerza para lograr este cambio (Torres Salcido et al., 2012); (Moragues-Faus y Morgan, 2015);(De Cunto et al., 2017). La construcción de la estrategia se fundamenta en un nuevo marco de decisión público en el que los actores locales desarrollan e implementan la política alimentaria, con nuevos modelos y escalas de gobernanza. En su inicio es necesario el estudio y conocimiento del entorno agroalimentario local, seguido por la elaboración de un documento 
de diagnóstico y propuestas de actuación. Documento que, en la mayoría de las ciudades son sometidos a consulta y participación ciudadana antes de su validación definitiva.

Con esta perspectiva, el objeto de este trabajo es el de contribuir al diseño de procesos de Estrategias Agroalimentarias de carácter Local. Se propone un desarrollo en etapas del proceso de diseño y se aplica la propuesta a un municipio situado en el área metropolitana de la ciudad de Valencia.

\section{Metodología}

El proceso consta de tres fases de diseño y una fase de implementación.

- Fase 1. Análisis de la situación. Implica: a) identificación de los aspectos relevantes del ámbito geográfico y socioeconómico de aplicación de la estrategia; b) identificación de los agentes clave y sus interacciones c) determinar los planos y niveles de interacción entre los actores. La fase concluye con la elaboración de un análisis DAFO de situación.

- Fase 2. Identificación de retos agroalimentarios en el ámbito de la estrategia. Los retos se individualizan para cada uno de los actores identificados y se establece una escala de posibles prioridades. La fase se refuerza con encuestas a los ciudadanos y concluye con sesiones abiertas de participación ciudadana, incluidos los principales agentes de la estrategia, para el contraste de la escala de prioridades.

- Fase 3. Definición de líneas estratégicas de actuación. Esta fase conlleva el diseño del plan de implementación de la estrategia a través de la definición de los ejes prioritarios y las líneas de actuación que incluyen. La fase concluye con la periodificación y la determinación de indicadores de seguimiento y control. La programación abarca un período presupuestario de tres años con acciones ejecutables en un ejercicio económico.

- Fase 4. Ejecución de las acciones previstas y medición de resultados.

\section{Resultados}

Del análisis de los datos obtenidos en las fases de diseño fue posible identificar los retos alimentarios a los que se enfrentan el sector productor agrario, el comercio, la administración pública y los consumidores, siendo el fomento de la alimentación de proximidad el elemento clave sobre el que pivota la estrategia y el que permite la terciarización de la actividad productiva agraria a través de canales de comercio directo tanto a consumidores como a empresas de transformación. Mediante la realización de una encuesta ciudadana se ha querido obtener información sobre la actitud, conocimiento y posición de la ciudadanía respecto al establecimiento de redes de distribución alimentaria alternativas basadas en productos de origen local. Se diseñaron 21 preguntas y se recabaron 84 respuestas con un margen de error del 7\%. De la encuesta se pudo deducir el desconocimiento general sobre la potencialidad que ofrecen los sistemas agroalimentarios locales sostenibles para la seguridad alimentaria local. Asimismo, destaca la buena predisposición a la creación de una plataforma comercial online incluida o ligada a la página web del Ayuntamiento.

\section{Conclusiones}

En este trabajo se ha partido de la literatura académica preexistente para trazar el proceso de diseño de una estrategia agroalimentaria. Para ello se ha formulado una propuesta de modelo metodológico y se ha realizado una prospección para observar la viabilidad de su implantación en un municipio (Paiporta) integrado en la Huerta Sur del entorno metropolitano de la ciudad de Valencia. Si bien es cierto que a lo largo del tiempo el municipio de Paiporta ha perdido peso agrícola y la Huerta se ve muy amenazada por la dinámica urbana expansionista, lo cierto es que cuenta con importantes recursos potencialmente favorables para el desarrollo de un sistema agroalimentario local más sostenible vinculado con la agricultura. La implicación de la administración local en el proyecto confiere también dinamismo a la iniciativa si bien es imprescindible la dotación de un presupuesto específico para las acciones que se desean desarrollar, por lo que puede ser necesario la creación o potenciación de un organismo específico coordinador de las acciones. El despliegue de indicadores y el control de su ejecución y eficiencia es el elemento de cierre de la estrategia y el instrumento que permitiría su revisión y continuidad.

\section{Bibliografía}

Boucher, F. y Reyes González, J. (2016). El Enfoque SIAL como catalizador de la acción colectiva: casos territoriales en América Latina. Estudios Sociales: Revista de Investigación Científica, 25(47), 1137.

Brunori, G., Galli, F., Barjolle, D., van Broekhuizen, R., Colombo, L., Giampietro, M., Kirwan, J., Lang, 
T., Mathijs, E., Maye, D., de Roest, K., Rougoor, C., Schwarz, J., Schmitt, E., Smith, J., Stojanovic, Z., Tisenkopfs, T. y Touzard, J. M. (2016). Are local food chains more sustainable than global food chains? Considerations for Assessment. Sustainability (Switzerland), 8(5), 1-27. https://doi.org/10.3390/su8050449

Calori, A., Dansero, E., Pettenati, G. y Toldo, A. (2017). Urban food planning in Italian cities: a comparative analysis of the cases of Milan and Turin. Agroecology and Sustainable Food Systems, 41(8), 1026-1046. https://doi.org/10.1080/21683565.2017.1340918

Cerrada-Serra, P., Colombo, L., Ortiz-Miranda, D. y Grando, S. (2018). Access to agricultural land in periurban spaces: social mobilisation and institutional frameworks in Rome and Valencia. Food Security, 10, 1325-1336. https://doi.org/10.1007/s12571-018-0854-8

De Cunto, A., Tegoni, C., Sonnino, R. y Michel, C. (2017). Food in cities: Study on innovation for a sustaiable and healthy production, delivery, and consumption of food in cities (Issue July). https://ec.europa.eu/research/openvision/pdf/rise/food_in_cities.pdf

Donald, B., y Blay-Palmer, A. (2006). The urban creative-food economy: Producing food for the urban elite or social inclusion opportunity? Environment and Planning A, 38(10), 1901-1920. https://doi.org/10.1068/a37262

FAO. (2011). Food, Agriculture and Cities: Challenges of Food and Nutrition Security, Agriculture and Ecosystem Management in an Urbanizing World.

FAO y OPS. (2016). América Latina y el Caribe: Panorama de la seguridad alimentaria y nutricional. Sistemas alimentarios sostenibles para poner fin al hambre y la malnutrición, 2016. In Us1.1. www.paho.org

Gliessman, S. . R., Rosado-May, F. J., Guadarrama-Zugasti, C., Jedlicka, J., Cohn, A., Mendez, V. E., Cohen, R., Trujillo, L., Bacon, C, y Jaffe, R. (2007). Agroecología: promoviendo una transición hacia la sostenibilidad. $\quad$ Ecosistemas, $16((1)), \quad 13-23$. http://www.revistaecosistemas.net/articulo.asp?Id=459

Hinrichs, C. C. (2000). Embeddedness and local food systems: Notes on two types of direct agricultural market. Journal of Rural Studies, 16, 295-303. https://doi.org/10.1016/S0743-0167(99)00063-7

Kneafsey, M., Dowler, E., Lambie-Mumford, H., Inman, A. y Collier, R. (2013). Consumers and food security: Uncertain or empowered? Journal of Rural Studies, 29(2013), 101-112. https://doi.org/10.1016/j.jrurstud.2012.05.005

Marsden, T. y Sonnino, R. (2012). Human health and wellbeing and the sustainability of urban-regional food systems. In Current Opinion in Environmental Sustainability. https://doi.org/10.1016/j.cosust.2012.09.004

Mincyte, D. y Dobernig, K. (2016). Urban farming in the North American metropolis: Rethinking work and distance in alternative food networks. Environment and Planning A, 48(9), 1767-1786. https://doi.org/10.1177/0308518X16651444

Moragues-Faus, A. y Morgan, K. (2015). Reframing the foodscape: the emergent world of urban food policy. Environment and Planning A, 47(7), 1558-1573. https://doi.org/10.1177/0308518X15595754

Muchnik, J., Cañada, J. S. y Salcido, G. T. (2008). Systèmes agroalimentaires localisés: État des recherches et perspectives. Cahiers Agricultures, 17(6), 513-519. https://doi.org/10.1684/agr.2008.0251

Opitz, I., Specht, K., Piorr, A., Siebert, R. y Zasada, I. (2017). Effects of consumer-producer interactions in alternative food networks on consumers' learning about food and agriculture. Moravian Geographical Reports, 25(3), 181-191. https://doi.org/10.1515/mgr-2017-0016

Paül, V. y McKenzie, F. H. (2013). Peri-urban farmland conservation and development of alternative food networks: Insights from a case-study area in metropolitan Barcelona (Catalonia, Spain). Land Use Policy, 30(1), 94-105. https://doi.org/10.1016/j.landusepol.2012.02.009

Pribadi, D. O. y Pauleit, S. (2015). The dynamics of peri-urban agriculture during rapid urbanization of Jabodetabek Metropolitan Area. Land Use Policy, 48, 13-24. https://doi.org/10.1016/j.landusepol.2015.05.009

Rastoin, J. (2015). Les systèmes alimentaires territorialisés: considérations théoriques et justifications empiriques, éditoriales. Économies et Sociétés, 49, 1155-1166.

Renting, H., Marsden, T. K. y Banks, J. (2003). Understanding alternative food networks: Exploring the role of short food supply chains in rural development. Environment and Planning A, 35(3), 393-411. https://doi.org/10.1068/a3510

Rodríguez Guerra, V., Sanz-Cañada, J. y García Azcarate, T. (2017). Canales Cortos de Comercialización 
en Panamá: factores condicionantes de las iniciativas promotoras. Estudios Sociales. Revista de Alimentación Contemporánea y Desarrollo Regional, 27(50). https://doi.org/10.24836/es.v27i50.421

Sánchez, J. (2009). Redes Alimentarias Alternativas : Concepto, Tipología y Adecuación. Boletín de La Asociación de Geógrafos Españoles, 49, 185-208.

Torres-Salcido, G. y Sanz-Cañada, J. (2018). Territorial Governance. A Comparative Research of Local Agro-Food Systems in Mexico. Agriculture, 8(2), 18. https://doi.org/10.3390/agriculture8020018

Torres Salcido, G., Larroa Torres, R. M.. Universidad Nacional Autónoma de México. Centro de Investigaciones Interdisciplinarias en Ciencias y Humanidades. (2012). Sistemas agroalimentarios localizados: identidad territorial, construcción de capital social e instituciones. Colección Alternativas.

Zoll, F., Specht, K., Opitz, I., Siebert, R., Piorr, A. y Zasada, I. (2018). Individual choice or collective action? Exploring consumer motives for participating in alternative food networks. International Journal of Consumer Studies, 42(1), 101-110. https://doi.org/10.1111/ijcs.12405 